Original Paper http://ajol.info/index.php/ijbcs http://indexmedicus.afro.who.int

\title{
Pression anthropique et dynamique des peuplements de Isoberlinia doka Craib et Stapf et Isoberlinia tomentosa (Harms) Craib et Stapf dans le domaine soudanien du Burkina Faso
}

\author{
Zézouma SANON ${ }^{1,2 *}$, Jérôme T. YAMEOGO ${ }^{2}$, Habou RABIOU ${ }^{3}$ et Mipro HIEN ${ }^{2}$ \\ ${ }^{I}$ Institut de l'Environnement et de Recherches Agricoles/Département Environnement et Forêts (INERA/DEF) \\ ,03 BP 7047, Ouagadougou 03, Burkina Faso. \\ ${ }^{2}$ Université Nazi Boni, institut du développement rural (IDR), Département des Eaux et Forêts. 01 BP 1091 \\ Bobo-Dioulasso 01, Burkina Faso. \\ ${ }^{3}$ Département de Production Végétale, Faculté des Sciences Agronomiques, Université de Diffa, BP 78 Diffa, \\ Niger. \\ *Auteur correspondant ; E-mail : zezoumasanon@yahoo.fr
}

\section{RESUME}

L'objectif de cette étude était de déterminer l'influence des activités anthropiques incontrôlées ainsi que du type de végétation sur la structure des peuplements de Isoberlinia spp.. Les données ont été collectées dans 43 placeaux installés dans les forêts claires et les savanes arborées/boisées situées dans la forêt classée et hors forêt classée. Les paramètres considérés ont été le diamètre, la hauteur, la densité, la surface terrière et la valeur d'importance écologique. Le test non-paramétrique de Kruskal-Wallis et la distribution de Weibull ont permis respectivement de comparer les moyennes et d'apprécier la dynamique. Dans la forêt classée, les valeurs moyennes du diamètre, de la hauteur et de la surface terrière étaient plus élevées et différaient ( $\mathrm{p}<$ $0,01)$ de celles obtenues hors forêt classée. En revanche, la densité moyenne n'a pas variée significativement ( $p$ $>0,05)$ entre les deux entités. La densité de régénération est importante, mais peu s'affranchissent. Suivant le type de formation végétale, les forêts claires sont dominées par des individus de gros diamètres et de grande taille. La structure de Isoberlinia spp. présente une allure en cloche avec le paramètre de weibull $\mathrm{c}=2,32$, traduisant une distribution asymétrique positive avec une prédominance d'individus de gros diamètre. Les peuplements de Isoberlinia spp. sont mieux conservés dans la forêt classée, notamment dans les forêts claires. Cependant, la faible capacité de l'espèce à assurer la succession des classes menace la survie de ces peuplements. La prise de mesures de gestion appropriées alors s'impose.

(C) 2019 International Formulae Group. All rights reserved

Mots clés: Dégradation, peuplements, structure, forêt classée de Koulbi, forêts claires.

\section{Effects of anthropogenic pressure on stands of Isoberlinia doka Craib and Stapf and Isoberlinia tomentosa (Harms) Craib and Stapf in the Sudanian zone of Burkina Faso}

\begin{abstract}
The objective of this study was to determine the influence of uncontrolled human activities and vegetation type on the stand structure of Isoberlinia spp. Data were collected in 43 plots installed in woodland, tree and wood savannah stands located inside the protected and unprotected areas. Parameters considered were
\end{abstract}


stem diameter, tree height, stand density, basal area and importance value index. The non-parametric KruskalWallis test was used to compare averages. The Weibull distribution was used to assess the structure dynamic of stands inside and outside the protected area. In the protected area, the mean values of diameter, height and basal area were significantly higher $(\mathrm{p}<0.01)$ compared to those observed outside the classified forest. In contrast, mean stand density was not significantly different $(\mathrm{p}>0.05)$ between the groups. The density of the regeneration is quite important. But most of them (75\%) remain in the height class] 0 to $0.5 \mathrm{~m}]$. According to vegetation types, woodland are dominated by large individuals. The structure of Isoberlinia spp. has a bell shaped curve with the weibull parameter $\mathrm{c}=2.32$, reflecting a positive asymmetric distribution with a predominance of large diameter individuals. The stands of Isoberlinia spp. are better conserved in the classified forest, especially in open forests. The low regeneration capacity threatens the survival of these stands in the long term. So better management measures are therefore needed to halt the degradation process.

(C) 2019 International Formulae Group. All rights reserved

Keywords: Degradation stands structure, classified forest of Koulbi, woodland.

\section{INTRODUCTION}

La répartition du genre Isoberlinia en Afrique de $1^{\prime}$ Ouest se situe entre $8^{\circ}$ et $13^{\circ}$ de latitude nord (White, 1986). Ce genre est représenté par deux espèces qui sont Isoberlinia doka et Isoberlinia tomentosa caractéristiques des forêts claires du domaine soudanien (Fontes et Guinko, 1995 ; Traoré, 2013). Les forêts claires à Isoberlinia spp. seraient issues de la dégradation des forêts sèches (Fournier, 1991). Elles jouent divers rôles tels qu'écologique, agricole, économique et vital pour les populations riveraines (Dourma et al., 2009a). Mais leur survie est mise en mal par la péjoration climatique et la pression anthropique. Dourma et al. (2012) notent que la pression anthropique aussi bien dans les terroirs villageois que dans les zones de conservation a entrainé une dégradation de la structure de Isoberlina spp. ainsi que de son habitat. Cette pression se traduit par l'expansion $\mathrm{du}$ front agricole et la surexploitation du bois d'œuvre (Glèlè-Kakaï et Sinsin, 2009).

Au Burkina Faso, les peuplements de Isoberlinia spp se rencontrent dans le secteur sud-soudanien (Boussim, 2010). Ces peuplements s'étendent par endroit sur plusieurs kilomètres et impriment une physionomie particulière aux formations végétales de la zone. Depuis les sécheresses des années 80, la partie Ouest du Burkina Faso est très convoitée par des migrants agricoles et agro-pasteurs entrainant une transformation rapide du paysage (Ouédraogo et al., 2010). Les aires protégées sont les sanctuaires de la conservation des écosystèmes et de la diversité biologique. De nos jours, les activités humaines illégales sont menaçantes pour une gestion durable de ces aires protégées (Tankoano et al., 2015 ; Belem et al., 2018). Dans la forêt classée de Koulbi et ses environs, cette pression sur les ressources naturelles s'est exacerbée avec le retour massif des Burkinabè à la suite de crise ivoirienne de 2002 (Courtin et al., 2010). A cela s'ajoutent les feux de brousse qui affectent la régénération naturelle des espèces ligneuses (Bationo et al., 2005). L'ensemble de ses facteurs ont un impact négatif sur la structure, la composition et la dynamique des espèces ligneuses (Gnoumou et al., 2012 ; Soulama et al., 2015a ; Abdourhamane et al., 2017).

Les peuplements de Isoberlinia spp. du Burkina Faso ne bénéficiant pas d'attention particulière de conservation, se caractérisent donc par très peu d'informations disponibles sur leur dynamique. D'où l'importance d'évaluer le potentiel existant. Le but de cette étude est d'améliorer les connaissances sur les peuplements de Isoberlinia spp. et d'aider à la prise de décisions quant à leur gestion durable. L'objectif était de déterminer l'influence des activités anthropiques incontrôlées sur la structure des peuplements de Isoberlinia spp. 
De façon spécifique, il s'est agi (i) d'analyser l'effet de la protection et du type de formation végétale sur la structure de Isoberlinia spp. (ii) et d'évaluer la régénération de Isoberlinia spp.

\section{METHODOLOGIE}

\section{Site d'étude}

La forêt classée de Koulbi (FCK) est située à l'extrême sud du Burkina Faso, entre les latitudes $9^{\circ} 29^{\prime}$ et $9^{\circ} 45^{\prime}$ Nord et les longitudes $2^{\circ} 42^{\prime}$ et $2^{\circ} 58^{\prime}$ Ouest, sur une superficie de 40000 ha (Figure 1). Elle appartient au domaine phytogéographique soudanien (White, 1986 ; Fontes et Guinko, 1995). Le climat est tropical sec caractérisé par une saison des pluies et une saison sèche (Boubacar, 2012). La pluviométrie et la température moyennes sur la période de 1971 à 2014 sont respectivement de $1057 \pm 149 \mathrm{~mm}$ et de $27,4 \pm 0,4{ }^{\circ} \mathrm{C}$ (Direction Générale de la Météo). Le fleuve Mouhoun constitue le principal cours d'eau. Les sols sont de types ferrugineux tropical, brunifié eutrophe et ferralitique. La végétation est dominée par les savanes (boisées, arborées et arbustives) auxquelles s'ajoutent les forêts galléries, les forêts claires (Fontes et Guinko, 1995 ; Sanon et al., 2015). La population de la province du Noumbiel était estimée à 94691 habitants en 2016 (INSD, 2017). Elle vit de l'agriculture extensive basée sur la production d'igname et la céréaliculture.

\section{Collecte de données}

L'inventaire a été fait dans des peuplements de Isoberlinia spp. suivant la technique d'échantillonnage aléatoire stratifié. Les strates considérées sont les savanes arborées, les savanes boisées et les forêts claires situées à l'intérieur et à l'extérieur de la forêt classée de Koulbi (FCK). La collecte de données a été faite dans une unité d'échantillonnage de circulaire de $900 \mathrm{~m}^{2}$, soit 23 placeaux dans la FCK et 20 hors de la forêt classée (HFC). Selon le type de formation végétale, 17 placeaux ont été installés dans les forêts claires (FC) et 26 dans les savanes arborées/boisées (SA/SB). A l'intérieur de chaque placeau, le diamètre et la hauteur de tous les individus dont le diamètre à $1,30 \mathrm{~m}$ du sol est supérieur à $5 \mathrm{~cm}$ ont été mesurés. La régénération a été collectée dans 215 placettes de $25 \mathrm{~m}^{2}$, puis répartie en classes de hauteur (m) :] 0-0,5] ;] 0,5-1 ] ;] 1-1,5 ] ;] 1,5-2 ] et > $2 \mathrm{~m}$ (Ouédraogo et al., 2009). La texture du sol, et la topographie du site ont été notées.

\section{Analyse des données}

La valeur moyenne des paramètres suivants a été calculée : la densité des sujets adultes $(\mathrm{N})$ et des juvéniles $\left(\mathrm{N}_{\mathrm{r}}\right)$, la surface terrière $(\mathrm{G})$, le diamètre $(\mathrm{D})$, la hauteur $(\mathrm{H})$ et la contribution $(\mathrm{Ci})$. $\mathrm{La}$ « $\mathrm{Ci}$ » en pourcentage, est définie comme étant la part de l'espèce dans la densité de l'ensemble de toutes les espèces. La richesse spécifique (S), l'indice de diversité de Shannon (H') et l'équitabilité de Piélou (Eq) ont été également calculés. Les formules utilisées sont résumées dans le Tableau 1.

La valeur d'importance écologique des espèces (IVI) a aussi été calculée. IVI est la somme de sa dominance relative (Domr), de sa densité relative (Dr) et de sa fréquence relative (Fr). Sa valeur renseigne sur l'importance de l'espèce dans une communauté végétale.

Le test non-paramétrique de comparaison de $\mathrm{k}$ échantillons de KruskalWallis au seuil de 5\% a permis de comparer les moyennes en utilisant le logiciel XLSTAT 2015.1.03.

La dynamique structurale de Isoberlinia spp a été étudiée à travers des histogrammes de distribution de classes de diamètres. La distribution de Weibull à trois paramètres $(\mathrm{a}, \mathrm{b}$ et $\mathrm{c})$ a été superposée à ces histogrammes. Une analyse log-linéaire a été exécutée à l'aide du logiciel SAS version 9.1 pour tester l'ajustement entre la structure observée et la distribution de Weibull. Le résultat du test d'ajustement entre les deux structures (fréquences théorique et observée) est accepté si la valeur de probabilité du test est supérieure à 0,05 . 


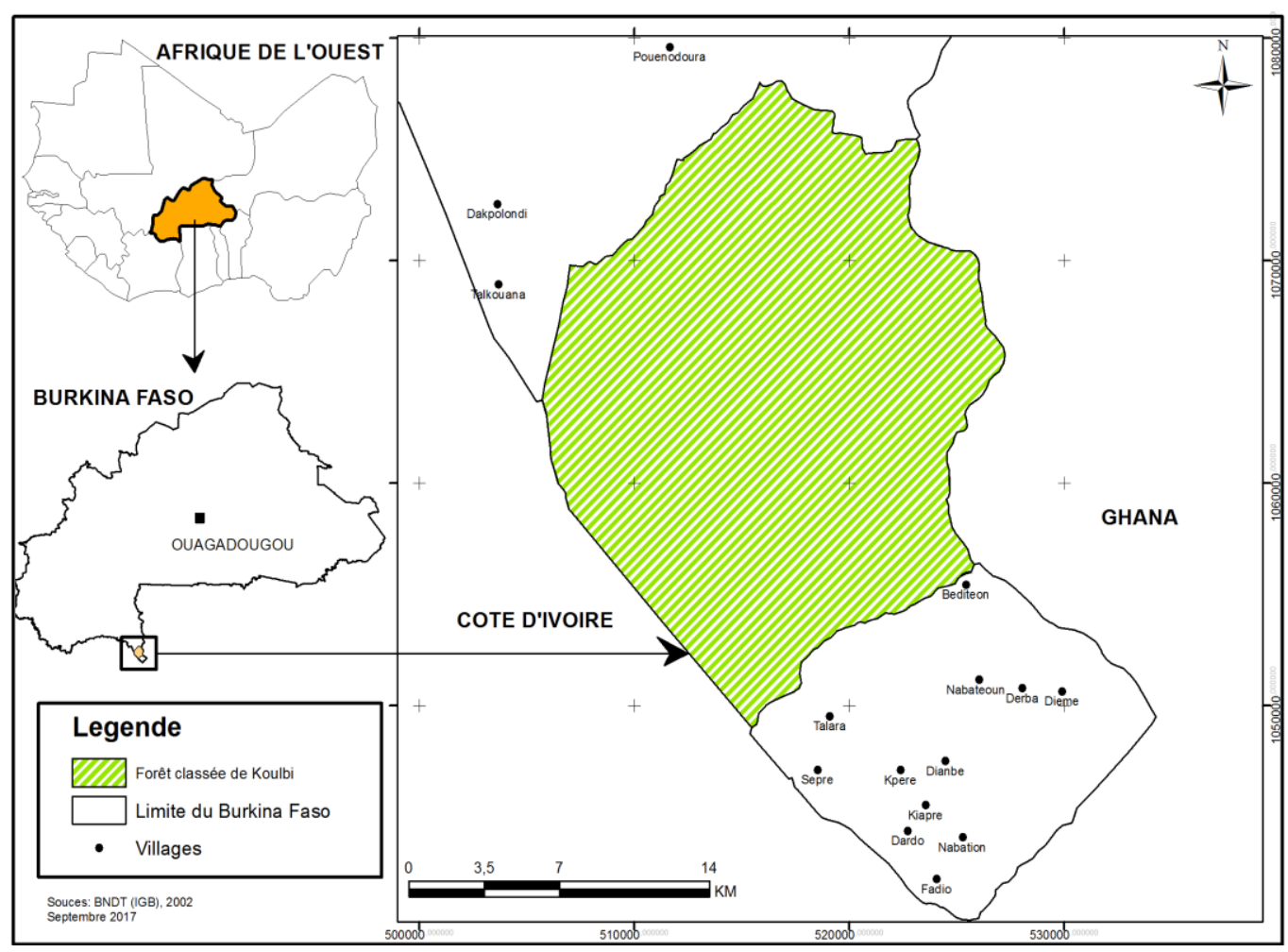

Figure 1 : Situation géographique de la zone d'étude.

Tableau 1: Synthèse des formules utilisées.

\begin{tabular}{|c|c|c|}
\hline Paramètres & Formules & $\begin{array}{l}\text { Signification des symboles } \\
\end{array}$ \\
\hline $\begin{array}{l}\text { Densité moyenne (N, nombre } \\
\text { sujets/ha) }\end{array}$ & $\mathrm{N}=\frac{\mathrm{n}}{\mathrm{s}}$ & $\begin{array}{l}\mathrm{n}=\text { nombre de sujets dans le placeau ; } \mathrm{s}: \text { aire } \\
\text { du placeau (ha). }\end{array}$ \\
\hline Surface terrière $\left(\mathrm{G}, \mathrm{m}^{2} / \mathrm{ha}\right)$ & $G=\frac{\pi}{4 s} \sum_{i=1}^{n} d i^{2}$ & $\begin{array}{l}\text { di : diamètre en } \mathrm{m} \text { de l'individu « } \mathrm{i} » ; \mathrm{n} \text { : } \\
\text { nombre d'individus, } \mathrm{s}: \text { aire du placeau (ha). }\end{array}$ \\
\hline $\begin{array}{l}\text { Densité régénération }\left(\mathrm{N}_{\mathrm{r}} \text {, nombre }\right. \\
\text { de plants/ha) }\end{array}$ & $\mathrm{N}_{\mathrm{r}}=\frac{\mathrm{n}_{\mathrm{r}}}{\mathrm{s}}$ & $\begin{array}{l}\mathrm{n}_{\mathrm{r}=} \text { nombre de plants, } \mathrm{s}=\text { aire de la placette } \\
\text { (ha) }\end{array}$ \\
\hline Contribution spécifique $\left(\mathrm{C}_{\mathrm{i}}, \%\right)$ & $C_{i}=\frac{X_{i}}{Y_{i}}$ & $\begin{array}{l}\mathrm{X}_{\mathrm{i}}=\text { valeur de Isoberlinia spp. considérant le } \\
\text { paramètre structural «i } \mathrm{i} » \\
\mathrm{Y}_{\mathrm{i}}=\text { valeur de toutes espèces par rapport au } \\
\text { paramètre structural «i } \mathrm{i} \text {. }\end{array}$ \\
\hline Indice de Shannon (H') & $\mathrm{H}^{\prime}=-\sum_{\mathrm{i}=1}^{\mathrm{S}} \mathrm{P}_{\mathrm{i}} \ln \mathrm{P}_{\mathrm{i}}$ & $\begin{array}{l}\mathrm{P}_{\mathrm{i}}: \mathrm{n}_{\mathrm{i}} / \mathrm{n} \text { où } \mathrm{n}_{\mathrm{i}}: \text { nombre d'individus espèce } \mathrm{i} \text { et } \\
\mathrm{n} \text { : nombre total d'individus, } \mathrm{S}: \text { richesse } \\
\text { spécifique. }\end{array}$ \\
\hline Indice de Pielou (Eq) & $\mathrm{Eq}=\frac{\mathrm{H}^{\prime}}{\ln S}$ & $\begin{array}{l}\mathrm{H}^{\prime}=\text { Indice de Shannon }\left(\mathrm{H}^{\prime}\right) \\
\text { lnS : logarithme népérien de la richesse } \\
\text { spécifique }\end{array}$ \\
\hline
\end{tabular}




\section{RESULTATS}

\section{Ecologie et diversité floristique des peuplements à Isoberlinia spp}

Les peuplements de Isoberlinia spp. colonisent les sols à texture de surface argilogravillonnaire, gravillonnaire et/ou rocailleuse bien drainés. On les retrouve sur les bas de pente, sur les versants et au sommet des collines. Dans la FCK, ils occupent la partie nord jusqu'à la limite de la rivière Koulbi au Sud. Ces peuplements sont par endroits entrecoupés par des plages de savanes.

Au total, 86 espèces ont été recensées. Elles se répartissent en 65 genres et 33 familles. Par ordre de valeur d'importance écologique (Tableau 2), on note Isoberlinia spp. $(56,36)$, Vitellaria paradoxa C.F. Gaertn (32,34), Lannea acida A. Rich. (20,56), Burkea africana Hook. (20,17), Pterocarpus erinaceus Poir. $(16,46)$, Combretum fragrans F. Hoffm. (11,53), Pseudocedrela kotschyi Harms $(10,47)$.

\section{Comparaison des paramètres suivant le niveau de protection}

Les paramètres écologiques (richesse spécifique, indice de Shannon, indice de Pielou) ne montrent pas de variation significative entre les peuplements de Isoberlinia spp. de la forêt classée de Koulbi et ceux situés hors de la forêt classée. Il en est de même de la densité des adultes et des juvéniles. Par contre, les paramètres dendrométriques qui montrent une différence significative entre ces deux entités sont le diamètre moyen, la surface terrière et la hauteur moyenne. Cela se vérifie tant avec toutes les espèces qu'avec seulement les individus de Isoberlinia spp. (Tableau 3). On ne note pas de contribution spécifique particulière de Isoberlinia spp. dans la constitution des densités aussi bien dans la forêt classée de Koulbi (16,08\%) que hors de la forêt classée $(15,70 \%)$.

\section{Comparaisons des paramètres suivant le type de formation végétale}

Les mêmes paramètres

dendrométriques et écologiques ont été comparées entre les deux types de formations végétales que sont les forêts claires et les savanes arborées/boisées (Tableau 4). Les densités des individus adultes et des régénérations ne présentent pas de différences significatives entre ces deux types de formations végétales. Les peuplements de Isoberlinia spp. situés dans les savanes sont écologiquement plus diversifiés que leur homologues situés dans les forêts claires. Par contre le diamètre moyen, la surface terrière et la hauteur moyenne des peuplements de Isoberlinia spp. des forêts claires, sont nettement plus élevés que ceux situés dans les savanes. Enfin, la contribution en densité des adultes de Isoberlinia spp. est plus élevée dans les forêts claires $(22,83 \%)$ que dans les savanes $(12,99 \%)$.

\section{Distribution par classe de diamètre des peuplements de Isoberlinia spp. \\ Structure suivant le niveau de protection}

Dans la forêt classée tout comme hors de la forêt classée, le peuplement dans son ensemble, présente une distribution en $~ « \mathbf{J}$ renversé » avec un paramètre $\mathrm{c}$ de weibull $<1$, caractéristique des peuplements multispécifiques ou inéquiennes (Figures $2 \mathrm{~A}$ et 2C). Par contre la structure en classe de diamètre de Isoberlinia spp. prise individuellement présente une distribution bimodale à l'intérieur de la forêt classée. Le premier mode se situe entre [5-15 cm [et le second entre [30-45 cm [. Cette distribution s'ajuste $(p>0,05)$ à une distribution théorique de Weibull avec un paramètre de forme $\mathrm{c}=$ 1,33. Ce qui indique que la structure obéit à une distribution asymétrique positive qui est caractéristique des peuplements monospécifiques à prédominance d'individus jeunes ou de faible diamètre (Figure 2B). La structure de la même espèce prise hors de la forêt classée présente une allure en «J renversé ». Cette distribution s'ajuste $(\mathrm{p}>$ $0,05)$ à une distribution théorique de Weibull avec un paramètre de forme $\mathrm{c}=0,87$, caractéristique des peuplements multispécifiques ou inéquiennes (Figure 2D).

\section{Structure suivant le type de formation}

Lorsque toutes les espèces sont prises en compte, la structure des peuplements à Isoberlinia spp. présente une distribution en « J » dans les forêts claires et les savanes arborées/boisées, caractéristique des 
peuplements multi-spécifiques avec dominance des sujets de petits diamètres (Figure 3A et 3C). Par contre, la structure de l'espèce Isoberlinia spp. présente des variations suivant le type de formation végétale. Dans les forêts claires, sa structure a une allure en cloche et s'ajuste à une distribution de weibull dont $\mathrm{c}=2,32$, traduisant une distribution asymétrique positive avec une prédominance d'individus adultes de diamètre compris entre 30 et $45 \mathrm{~cm}$ (Figure 3). Dans les savanes arborées/boisées, la structure de l'espèce est en $« \mathrm{~J} »$ avec le paramètre de forme $\mathrm{c}=0,93$, caractéristique des peuplements inéquiennes.
Structure de la régénération de Isoberlinia spp.

La structure en classe de hauteur de la régénération de Isoberlinia spp présente une distribution en « $\mathrm{L}$ ». Cette allure est la même suivant le niveau de protection et selon le type de formation végétale. L'essentiel de la régénération $(75 \%)$ est concentré dans la première classe de hauteur] 0-0,5 m] (Figure 4). Le processus naturel de régénération assure difficilement la dynamique des peuplements de Isoberlinia spp. dans la forêt classée de Koulbi et ses environs. On observe la même tendance lorsqu'on prend en compte la régénération de toutes les espèces ligneuses (Figure 5).

Tableau 2 : Valeur d'importance écologique des espèces.

\begin{tabular}{ll|ll}
\hline Espèces & IVI & Espèces & IVI \\
\hline Isoberlinia spp & $\mathbf{5 6 , 3 6}$ & Pterocarpus erinaceus & $\mathbf{1 6 , 4 6}$ \\
Vitellaria paradoxa & $\mathbf{3 2 , 3 4}$ & Combretum fragrans & $\mathbf{1 1 , 5 3}$ \\
Lannea acida & $\mathbf{2 0 , 5 6}$ & Pseudocedrela kotschyi & $\mathbf{1 0 , 4 7}$ \\
Burkea africana & $\mathbf{2 0 , 1 7}$ & Autres espèces & 137,23 \\
\hline IVI : valeur d'importance écologique.
\end{tabular}

Tableau 3: Paramètres structuraux des peuplements de Isoberlinia spp. suivant les niveaux de protection.

\begin{tabular}{|c|c|c|c|}
\hline Paramètres & FCK & HFC & P-value \\
\hline \multicolumn{4}{|l|}{ Toutes les espèces } \\
\hline Densité adulte (N, pieds/ha) & $384 \pm 181$ & $451 \pm 210$ & 0,71 \\
\hline Diamètre moyen $(\mathrm{D}, \mathrm{cm})$ & $15,18 \pm 9,8$ & $12,93 \pm 8,4$ & $0,01 *$ \\
\hline Surface terrière $(\mathrm{G}, \mathrm{m} 2 / \mathrm{ha})$ & $10,00 \pm 1,3$ & $8,44 \pm 1,5$ & $0,01 *$ \\
\hline Hauteur moyenne $(\mathrm{H}, \mathrm{m})$ & $7,1 \pm 2,9$ & $6,12 \pm 2,5$ & $0,01 *$ \\
\hline Densité juvénile (Nr plants/ha) & $7746 \pm 1068$ & $10723 \pm 3248$ & 0,89 \\
\hline \multicolumn{4}{|l|}{ Sujets de Isoberlinia spp. } \\
\hline Densité adulte $\left(\mathrm{N}_{\text {Iso }}\right.$, pieds/ha) & $57 \pm 43$ & $64 \pm 34$ & 0,80 \\
\hline Diamètre moyen $\left(\mathrm{D}_{\text {Iso, }} \mathrm{cm}\right)$ & $25,65 \pm 12,9$ & $18,16 \pm 12,4$ & $0,01 *$ \\
\hline Surface terrière $\left(\mathrm{G}_{\mathrm{Iso}}, \mathrm{m}^{2} / \mathrm{ha}\right)$ & $3,46 \pm 0,9$ & $1,82 \pm 1$ & $0,01 *$ \\
\hline Hauteur moyenne $\left(\mathrm{H}_{\mathrm{Iso}}, \mathrm{m}\right)$ & $10,26 \pm 3,3$ & $7,62 \pm 3,4$ & $0,01 *$ \\
\hline Densité juvénile $\left(\mathrm{Nr}_{\mathrm{Iso}}\right.$ plants/ha) & $513 \pm 153$ & $592 \pm 255$ & 0,73 \\
\hline Contribution densité adulte ( $\mathrm{Nc} \%)$ & $16,08 \pm 12$ & $15,70 \pm 11$ & 0,90 \\
\hline Contribution densité juvénile ( $\mathrm{Nr}_{\text {Iso }}$ plants/ha) & $7,56 \pm 10,4$ & $7,64 \pm 9,4$ & 0,85 \\
\hline \multicolumn{4}{|l|}{ Paramètres écologiques } \\
\hline Richesse spécifique/relévé & $11 \pm 04$ & $12 \pm 03$ & 0,73 \\
\hline Richesse spécifique totale & 57 & 56 & ----- \\
\hline Indice de Shannon $(\mathrm{H})$ & 3,10 & 3,30 & ----- \\
\hline Indice d'équitabilité de Piélou (Eq) & 0,76 & 0,82 & ----- \\
\hline
\end{tabular}

FCK : Forêt Classée de Koulbi ; HFC : Hors Forêt Classée ; *: différence significative. 
Tableau 4: Paramètres structurales de Isoberlinia spp. suivant le type de formation végétale.

\begin{tabular}{|c|c|c|c|}
\hline Paramètres & FC & SA/SB & p-value \\
\hline \multicolumn{4}{|l|}{ Toutes les espèces } \\
\hline Densité adulte (N, pieds/ha) & $332 \pm 70$ & $436 \pm 223$ & 0,13 \\
\hline Diamètre moyen $(\mathrm{D}, \mathrm{cm})$ & $18,20 \pm 10,9$ & $13,17 \pm 8,5$ & $0,01 *$ \\
\hline Surface terrière $(\mathrm{G}, \mathrm{m} 2 / \mathrm{ha})$ & $11,77 \pm 1,2$ & $8,39 \pm 1,4$ & $0,01 *$ \\
\hline Hauteur totale moyenne $(\mathrm{H}, \mathrm{m})$ & $8,00 \pm 3,5$ & $6,35 \pm 2,4$ & $0,01 *$ \\
\hline Densité juvénile (Nr plants/ha) & $5769 \pm 791$ & $9893 \pm 1684$ & 0,32 \\
\hline \multicolumn{4}{|l|}{ Sujets de Isoberlinia spp. } \\
\hline Densité adulte $\left(\mathrm{N}_{\text {Iso }}\right.$, pieds/ha) & $74 \pm 36$ & $52 \pm 42$ & 0,06 \\
\hline Diamètre moyen $\left(\mathrm{D}_{\text {Iso, }} \mathrm{cm}\right)$ & $29,98 \pm 10,9$ & $17,95 \pm 12,2$ & $0,01^{*}$ \\
\hline Surface terrière $\left(\mathrm{G}_{\mathrm{Iso}}, \mathrm{m}^{2} / \mathrm{ha}\right)$ & $5,94 \pm 0,6$ & $1,87 \pm 1,3$ & $0,01 *$ \\
\hline Hauteur moyenne $\left(\mathrm{H}_{\mathrm{Iso}}, \mathrm{m}\right)$ & $11,78 \pm 2,9$ & $8,11 \pm 3,1$ & $0,01 *$ \\
\hline Densité juvénile $\left(\mathrm{Nr}_{\text {Iso }}\right.$ plants/ha) & $669 \pm 308$ & $480 \pm 134$ & 0,72 \\
\hline Contribution densité adulte $\left(\mathrm{Nc}_{\text {Iso }} \%\right)$ & $22,83 \pm 1$ & $12,99 \pm 10,6$ & $0,04 *$ \\
\hline Contribution densité juvénile $\left(\mathrm{Nrc}_{\text {Iso }} \%\right)$ & $9,14 \pm 11,6$ & $6,91 \pm 9,4$ & 0,72 \\
\hline \multicolumn{4}{|l|}{ Paramètres écologiques } \\
\hline Richesse spécifique/relevé & $10 \pm 02$ & $12 \pm 04$ & 0,08 \\
\hline Richesse spécifique totale & 36 & 69 & ----- \\
\hline Indice de Shannon $(\mathrm{H})$ & 2,75 & 3,38 & ----- \\
\hline Indice d'équitabilité de Piélou (Eq) & 0,55 & 0,77 & ----- \\
\hline
\end{tabular}

FC = Forêts Claires à Isoberlinia spp, SA/SB = Savanes Arborées/Boisées à Isoberlinia spp.; *: différence significative.

Forêt Cassée de Koulbi (FCK)
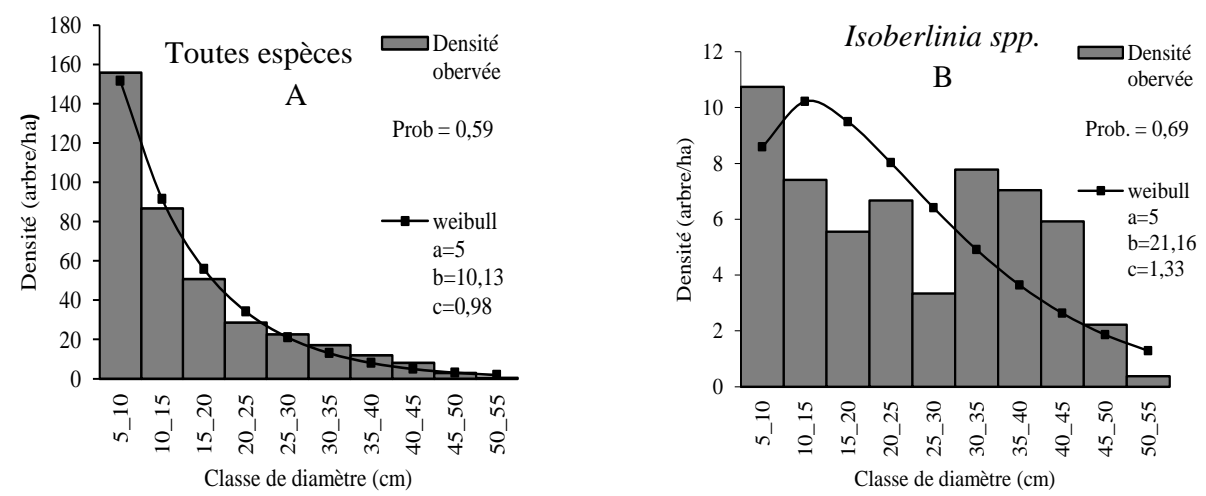

Hors de la Forêt Classée (HFC)
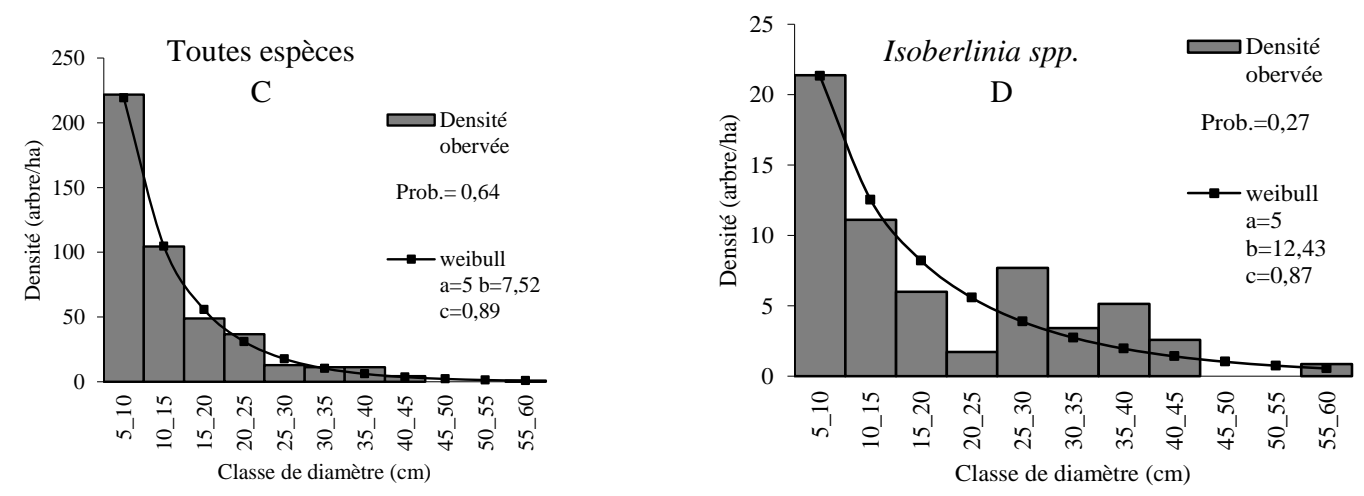

Figure 2 : Distribution par classe de diamètre de Isoberlinia spp. suivant le statut de conservation. 
Forêts claires à Isoberlinia spp. (FC)
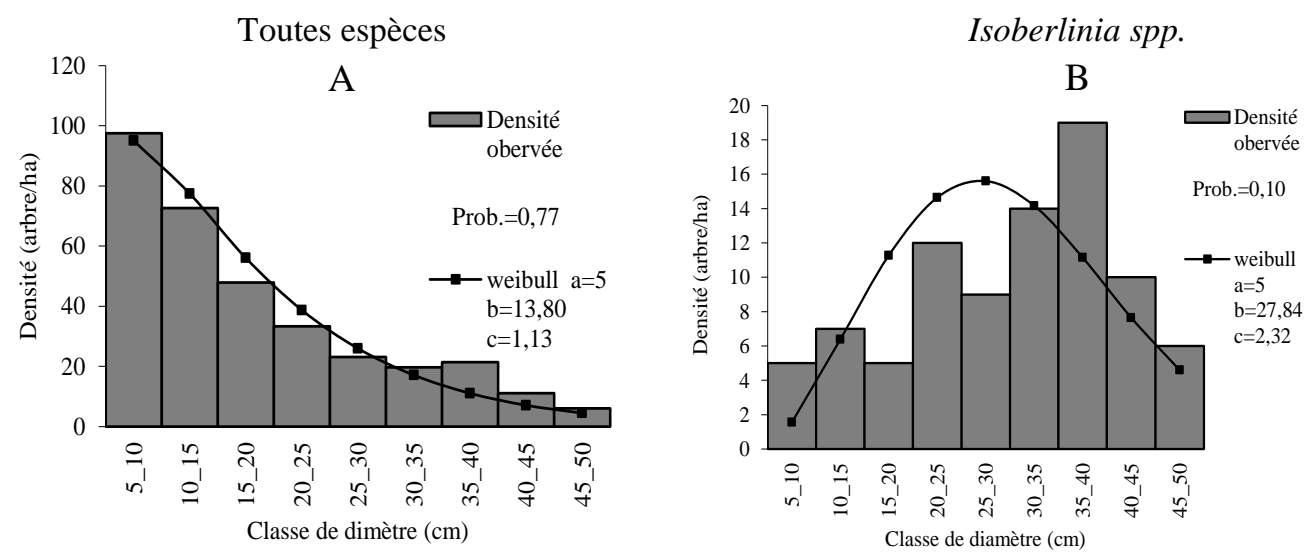

Savanes arborées/boisées à Isoberlinia spp. (SA/SB)
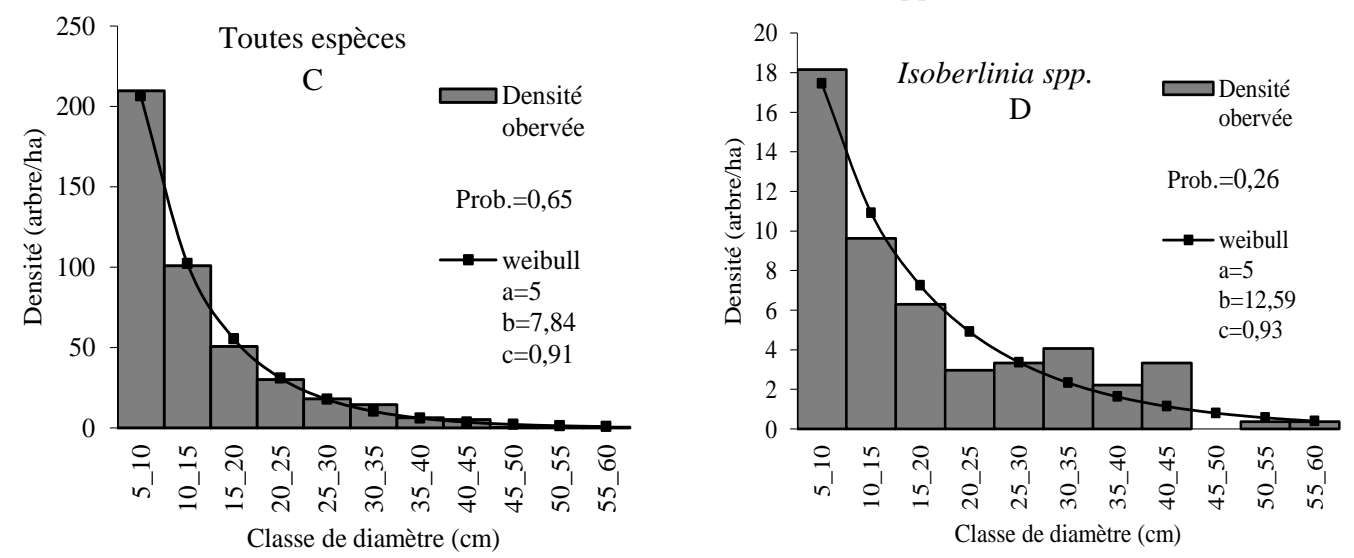

Figure 3: Distribution par classe de diamètre de Isoberlinia spp. suivant le type de formations végétales.

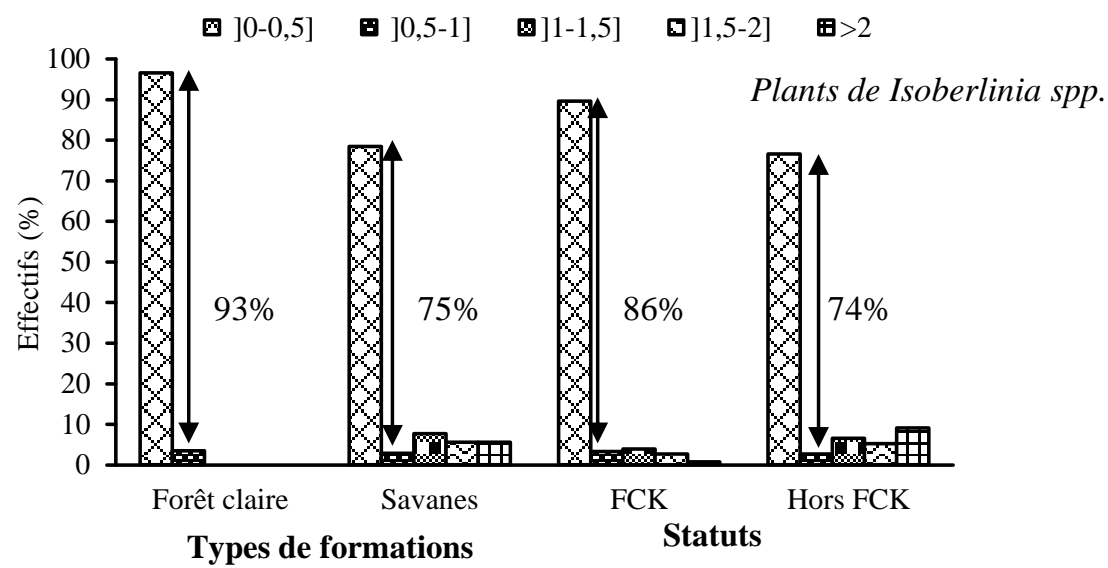

Figure 4: Distribution par strates de hauteur des plants de Isoberlinia spp. 


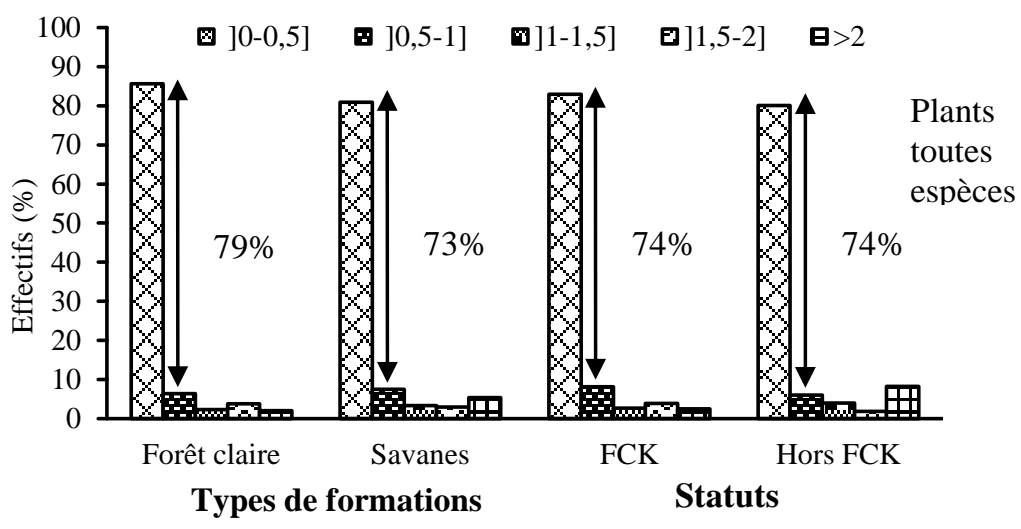

Figure 5: Distribution par strates de hauteur de la régénération des peuplements de Isoberlinia spp.

\section{DISCUSSION}

\section{Diversité floristique des peuplements de Isoberlinia spp.}

Les peuplements de Isoberlinia spp. sont moyennement diversifiés. La richesse spécifique de notre zone d'étude est plus élevée que celle obtenue par Dourma et al. (2009a). Suivant l'influence du statut de protection sur la diversité floristique ligneuse des peuplements à Isoberlinia spp., les mêmes auteurs démontraient que la richesse spécifique variait peu selon que l'on soit dans une forêt protégée ( 31 espèces) ou hors de la forêt protégée (28 espèces). Ce qui est similaire à notre constat dans les peuplements de Isoberlinia spp. étudiés. Les auteurs Gnoumou et al. (2012) et Assogbadjo et al. (2009) sont arrivés à la même conclusion respectivement sur les peuplements grégaires de Guibourtia copallifera Benn. et Anogeissus leiocarpa Guill., Perr. Cela suggère que la perturbation affecte peu la diversité floristique des peuplements de Isoberlinia spp.

Les peuplements de Isoberlinia spp. situés dans les savanes sont écologiquement plus diversifiés que leur homologues situés dans les forêts claires. La forte hétérogénéité spécifique des savanes arborées/boisées de
Isoberlinia spp. traduit un état de perturbation moyenne comme l'explique "l'hypothèse de la perturbation intermédiaire". Selon ce principe, dans la succession végétale, la richesse spécifique est plus élevée dans l'état intermédiaire que dans l'état final. Les perturbations favoriseraient la coexistence des espèces en créant une hétérogénéité spatiale et temporelle des dynamiques de successions. Cette hétérogénéité permet aux espèces fugitives de réaliser leur cycle de développement en dépit de la présence de compétiteurs (Cordonnier, 2004). Les savanes arborées/boisées de Isoberlinia spp. seraient alors une étape intermédiaire dans la dynamique de successions vers les forêts claires, elles même nées de la perturbation des forêts sèches (Fournier, 1991).

Par ailleurs, la comparaison de nos résultats, avec ceux de Dourma et al. (2009a) au nord du Togo et avec ceux de Goussanou et al. (2017) au centre du Bénin, études conduites respectivement autour de $9^{\circ}, 8^{\circ}$ et $7^{\circ}$ de latitudes nord, montre que lorsqu'on descend les latitudes, la richesse spécifique des peuplements de Isoberlinia spp. diminue. L'augmentation de la pluviométrie favoriserait le développement des peuplements de Isoberlinia spp. tendant vers 
de peuplements plus homogènes, réduisant ainsi la richesse spécifique ligneuse en leur sein.

\section{Structure et dynamique des peuplements de Isoberlinia spp.}

L'analyse

des paramètres

dendrométriques révèle que les plus gros diamètres et les plus grandes hauteurs de Isoberlinia spp. ont été relevés dans la forêt classée de Koulbi comparativement à la partie hors forêt. Cela montre que la protection améliore la structure des peuplements naturels comme l'ont constaté Assogbadjo et al. (2009); Gnoumou et al. (2012) et Abdourhamane et al. (2017) respectivement sur les peuplements de Anogeissus leiocarpa Guill., Perr., de Guibourtia copallifera Benn. et de Sclerocarya birrea (A.Rich) Hochst. Par contre, cette protection n'affecte pas significativement la densité des sujets adultes et celle de la régénération. Traoré et al. (2013) ont obtenu un résultat similaire lors de leurs travaux sur la régénération de Diospyros mespiliformis Hochst. ex A. DC., de Prosopis africana (G. et Perr.) Taub. et de Sterculia setigera Del. Le gradient d'anthropisation semble alors avoir peu d'effet sur la densité des peuplements naturels (Soulama et al., 2015b). Les aires protégées sont assujetties aux feux de brousse et aux pâtures au même titre que les aires non protégées. En effet, l'intensité des feux de brousse dans les formations tropicales est telle que peu de jeunes plants survivent après leurs passages (Palumbo et al., 2011 ; Doamba et al., 2014). Selon Bationo et al. (2005), seuls 30\% des plantules de Isoberlinia spp. de la première année ont la chance de survivre après les feux de brousse. Ce phénomène expliquerait l'allure en « $\mathrm{L}$ » de la distribution en classes de hauteur de la régénération. Cette contrainte de régénération se répercute sur la distribution en classes de diamètre de l'espèce aussi bien dans la forêt classée que hors de la forêt classée. La structure de Isoberlinia spp. en « J renversé », notée hors de la forêt classée, ne traduirait pas une bonne dynamique de l'espèce dans cette partie. L'absence d'individus dans les classes de diamètres ] 45$50 \mathrm{~cm}$ ] et ] 50-55 cm] traduit une pression sélective sur l'espèce hors de la forêt classée. La pression anthropique (feux de brousse, pâturage, coupe sélective de Isoberlinia spp.) serait donc la cause de la perturbation affectant la régénération de ces espèces. Ces résultats corroborent ceux de Assogbadjo et al. (2009) sur la structure de Anogeissus leiocarpa (DC.) Guill. et Perr. Ils soutiennent que dans une situation de pression anthropique incontrôlée, le prélèvement tend à être dirigé contre les individus de gros diamètres. Isoberlinia spp. exploitée comme bois d'œuvre ou bois de service (Dourma et al., 2009a) serait sujette à une surexploitation $\mathrm{du}$ fait de la raréfaction des bois d'œuvre de prédilection que sont Milicia excelsa (Welw.) C.C.Berg, Afzelia africana Sm. et Khaya senegalensis (Desr.) A.Juss. (Glèlè-Kakaï et Sinsin, 2010). Un autre facteur qui mettrait en péril les peuplements de Isoberlinia spp. serait la production d'igname basée sur l'agriculture itinérante où le plus souvent les producteurs défrichent chaque année un nouveau champ.

L'analyse des caractéristiques dendrométriques et structurales suivant le type de formation, montre que les forêts claires abritent les sujets de Isoberlinia spp. ayant les plus gros diamètres et la plus grande taille. La structure obéit à une allure en cloche avec une prédominance des individus de grand diamètre. La faible représentativité des individus de petits diamètres montre que les forêts claires assurent difficilement la dynamique normale des peuplements de Isoberlinia spp. Ces résultats corroborent ceux de Traore (2013) sur la même espèce dans le secteur sud-soudanien du Burkina Faso. Les 
forêts claires abritent des individus de gros diamètres qui sont des potentiels semenciers qui par autochorie dissémine les graines de Isoberlinia spp sous leur houppier (Bationo et al., 2005 ; Dourma et al., 2009b). La densité de la régénération plus importante dans les forêts claires que dans les savanes arborées/boisées pourrait s'expliquer alors par cette situation. Toutefois, cette régénération parvient difficilement à s'affranchir des feux de brousse, du pâturage et d'autres facteurs de dégradation. Ce qui se traduit par une distribution en classe de hauteur sous forme de $« \mathrm{~L} »$. Dans les forêts claires $93 \%$ de la régénération reste dans la première classe de hauteur et aucun plant dont la hauteur est supérieure à $1 \mathrm{~m}$ n'a été recensé. Ces plants qui, pour la plupart seraient issus de la régénération séminale sont plus sensibles aux feux de brousse que les drageons et les rejets de souches.

\section{Conclusion}

Cette étude visait à contribuer à une meilleure connaissance des peuplements de Isoberlinia spp. caractéristiques de la végétation dans la forêt classée de Koulbi et ses environs. Les résultats montrent qu'au plan écologique, les peuplements de Isoberlinia spp. sont perturbées avec la transition des forêts claires vers les savanes. Le diamètre moyen, la hauteur moyenne et la surface terrière de Isoberlinia spp. diffèrent significativement selon le niveau de protection et suivant le type de formation végétale. En revanche, la densité des individus adultes et juvéniles de Isoberlinia spp ne différe pas significativement suivant le type de formation et selon le niveau de protection. La structure en classes de diamètre présente une distribution asymétrique positive avec dominance des individus de gros diamètres dans la forêt classée et dans les forêts claires. L'allure en «J renversé » notée hors de la forêt classée et dans les savanes arborées/boisées serait le résultat de coupes sélectives sur des classes de diamètres donnés. La densité de la régénération de Isoberlinia spp. est assez élevée, mais plus de $75 \%$ de cette régénération restent dans la première classe de hauteur de] 0-0,5 m]. Les structures observées témoignent du problème lié à l'insuffisance de relais entre les plantules de Isoberlinia spp. et les jeunes individus d'une part et entre les jeunes individus et les individus adultes d'autre part. Il résulte que le processus naturel assure difficilement la régénération de l'espèce. La prise de mesures s'avère nécessaire pour sensibiliser les populations riveraines de la forêt classée de Koulbi sur la nécessité de préservation de Isoberlinia spp et de son habitat.

\section{CONFLITS D'INTERETS}

Les auteurs déclarent qu'ils n'ont pas de conflits d'intérêts.

\section{CONTRIBUTIONS DES AUTEURS}

ZS a coordonné les travaux de terrain et est le rédacteur principal de cet article. HR a analysé les données de végétation suivant la méthode de Weibull. JTY et MH ont donné les grandes orientations de la méthodologie de collecte de données et $\mathrm{MH}$ a appuyé à l'identification des espèces.

\section{REMERCIEMENTS}

Le projet UNDESERT (Understanding and combating desertification to mitigate its impact on ecosystem)/ UE-Financement. La direction provinciale de l'Environnement et des Ressources Halieutiques du Noubiel.

\section{REFERENCES}

Abdourhamane H, Rabiou H, Diouf A, Morou B, Mahamane A, Bellefontaine R. 2017. Structure démographique et répartition spatiale des populations de Sclerocarya 
birrea (A. Rich.) Hochst. du secteur sahélien du Niger. Bois et Forêts des Tropiques 333(3) : 55-66. DOI: https://doi.org/10.19182/bft2017.333.a31 468

Assogbadjo AE, Glèlè-Kakaï R, Sinsin B, Pelz D. 2009. Structure of Anogeissus leiocarpa Guill. Perr. natural stands in relation to anthropogenic pressure within Wari-Maro Forest Reserve in Benin. Afr.J. Ecol. 48: 644-653. https://doi.org/10.1111/j.1365-

2028.2009.01160.x

Bationo BA, Ouédraogo SJ, Some NA, Pallo F, Boussim IJ. 2005. Ecologie de la régénération naturelle de Isoberlinia doka Craib. et stapf. dans la forêt classée de Nazinon (Burkina Faso). Cah. Agric. 14(3) : 1-9. ISSN 1166-7699 Publié sous licence CC-By-NC.

Belem M. Zoungrana M. Nabaloum M. 2018. Les effets combinés du climat et des pressions anthropiques sur la forêt classée de Toéssin, Burkina Faso. Int. J. Biol. Chem. Sci., 12(5) : 2186-2201. DOI:

https://dx.doi.org/10.4314/ijbcs.v12i5.20

Boubacar I. 2012. Caractérisation des saisons de pluies au Burkina Faso dans un contexte de changement climatique et évaluation des impacts hydrologiques sur le bassin du Nakanbé. Hydrologie. Université Pierre et Marie Curie - Paris VI, 2012. Français. $\langle\mathrm{NNT}$ : 2012PA066087>. 〈tel-00827764>.

Boussim JI. 2010. Les territoires phytogéographiques. In A. Thiombiano \& D. Kampmann (Eds.), Biodiversity atlas of West Africa, Volume II: Burkina Faso; 152-155. Ouagadougou \& Frankfurt/Main.

Cordonnier T. 2004. Disturbance, diversity and permanence of structures in forest ecosystems. Life Sciences [q-bio].
ENGREF (AgroParisTech), 2004. English. <NNT: 04ENGR0010>. $<$ pastel00000909>.

Courtin F, Fournet F, Solano P. 2010. La crise ivoirienne et les migrants burkinabés: L'effet boomerang d'une migration internationale. Afrique contemporaine 236(4) : 11-27. DOI : https://doi.org/10.3917/afco.236.0011

Doamba SWMF, Savadogo P, Nacro HB. 2014. Rôle des feux de savane sur les caractéristiques biogéochimiques des sols en zone soudanienne du Burkina Faso. Int. J. Biol. Chem. Sci., 8(2) : 77793.

DOI : http://dx.doi.org/10.4314/ijbcs.v8i2.34

Dourma M, Batawila K, Wala K, Kokou K, Guelly AK, Bellefontaine R, De Foucault B, Akpagana K. 2009b. Régénération naturelle des peuplements de Isoberlinia en zone soudanienne du Togo, Afrique de l'Ouest. Acta Botanica Gallica, 156(3) : 415-425. DOI : http://dx.doi.org/10.1080/12538078.2009 .10516167

Dourma M, Wala K, Bellefontaine R, Batawila K, Guelly AK, Akpagana K. 2009a. Comparaison de l'utilisation des ressources forestières et de la régénération entre deux types de forêts claires à Isoberlinia au Togo. Bois et Forêt des Tropiques, 302(4) : 5-19. DOI: https://doi.org/10.19182/bft2009.302.a20 400

Dourma M, Wala K, Guelly KA, Bellefontaine R, Deleporte P, Akpavi S, Batawila K, Akpagana K. 2012. Typologie, caractéristiques structurales et dynamique des faciès forestiers fragiles à Isoberlinia spp. en vue de leur gestion au Togo. Bois et Forêts des Tropiques, 313(3) : 19-33. DOI: https://doi.org/10.19182/bft2012.313.a20 494 
Fontes J, Guinko S. 1995. Carte de la végétation et de l'occupation des sols du Burkina Faso. Notice explicative. Ministère coopération française, projet Campus (8813101). Toulouse: Université Paul Sabatier.

Fournier A. 1991. Phénologie, Croissance et production végétales dans quelques savanes d'Afrique de l'ouest, variation selon un gradient climatique. Editions de I'ORSTOM, Institut Français de Recherche Scientifique pour le Développement en Coopération, Collection Études et Thèses, PARIS, 312 p.

Glèlè-Kakaï R, Sinsin B. 2009. Structural description of two Isoberlinia dominated vegetation types in the Wari-Maro Forest Reserve (Benin). South African Journal of Botany, 75(1): 43-51. https://doi.org/10.1016/j.sajb.2008.07.00 3

Glèlè-Kakaï R, Sinsin B. 2010. Description de Isoberlinia spp Caesalpiniaceae. In book: Biodiversity Atlas of West Africa Vol 1.Edition: $\mathrm{N}^{\circ} 1$, Chapter: 6.13, Publisher: BIOTA. Editors: Brice Sinsin, Dorothea Kampmann.

https://www.researchgate.net/publication / 23390379. [accessed Aug 22 2018].

Gnoumou A, Bognounou F, Hahn K, Thiombiano A. 2012. A comparison of Guibourtia copallifera Benn. Stands in South West Burkina Faso-community structure and regeneration. Journal of Forestry Research 23 (1): 29-38. DOI 10.1007/s11676-011-0198-0

Goussanou CA, Tenté BA, Akouehou G, Salako VK, Romain L. Glèlè-Kakaï RL, Sinsin BA. 2017. Structural and spatial patterns of Isoberlinia species in a disturbed community forest (Benin, West Africa). Journal of Forestry
Faculty, 17(2) : 225-237. DOI: 10.17475/kastorman.340150.

INSD. 2017. Institut national de la statistique et de la démographie: Annuaire statistique 2016. Ministère de l'économie et des finances, Burkina Faso.

Ouédraogo I, Tigabu M, Savadogo P, Compaoré H, Odén PC, Ouadba J-M. 2010. Land cover change and its relation with population dynamics in Burkina Faso, West Africa. Land Degrad. Develop., 21: 453-462. DOI: https://doi.org/10.1002/ldr.981

Ouédraogo $\mathrm{O}$, Thiombiano A, Hahn-Hadjali K, Guinko S. 2009. Diversité et dynamique de la végétation ligneuse juvénile du Parc National d'Arly (Burkina Faso). Candollea, 64 : 257-278.

Ouoba P. 2006. Flore et végétation de la forêt classée de Niangoloko, sud-ouest du Burkina Faso. Thèse de Doctorat, Université de Ouagadougou. Burkina Faso. 144p.

Palumbo I, Grégoire J-M, Simonetti D, Punga M. 2011. Spatio-temporal distribution of fire activity in protected areas of SubSaharan Africa derived from MODIS data. Procedia Environmental Sciences, 7 : 26-31. DOI : https://doi.org/10.1016/j.proenv.2011.07. 006

Sanon Z, Hien M, Yaméogo JT, Bachmann Y, Somda I. 2015. Dynamique structurale des îlots de forêt claire à Anogeissus leiocarpa (DC.) Guill. et Perr. dans le Sud-Ouest du Burkina Faso. Int. J. Biol. Chem. Sci., 9(2): 847-860. DOI: http://dx.doi.org/10.4314/ijbcs.v9i2.24

Soulama S, Kadeba A, Nacoulma BMI, Savadogo P, Bachmann Y, Thiombiano A. 2015b. Do floristic composition, plant species abundance and vegetation structure in Sudanian wetlands vary according to conservation status? Global 
Journal of Wood Science, Forestry and Wildlife, 3(3): 083-093.

Soulama S, Kadeba A, Nacoulma BMI, Traoré S, Bachmann Y, Thiombiano A. 2015a. Impact des activités anthropiques sur la dynamique de la végétation de la réserve partielle de faune de Pama et de ses périphéries (sud-est du Burkina Faso) dans un contexte de variabilité climatique. J. Appl. Biosci., 87 : 80478064. DOI :

http://dx.doi.org/10.4314/jab.v87i1.6

Tankoano B, Hien M, Dibi NH, Sanon Z, Yaméogo JT, SomdaI. 2015. Dynamique spatio-temporelle des savanes boisées de la forêt classée de Tiogo au Burkina Faso, Int. J. Biol. Chem. Sci., 4(9): 19842000.

DOI:

http://dx.doi.org/10.4314/ijbcs.v9i4.23
Traoré L, Sop TK, Dayamba SD, Traoré S, Hahn K, Thiombiano A. 2013. Do protected areas really work to conserve species? A case study of three vulnerable woody species in the Sudanian zone of Burkina Faso. Environment Development and Sustainability, 15(3) : 663-686.

Traoré L. 2013. Influence du climat et de la protection sur la végétation ligneuse de la partie Occidentale du Burkina Faso, thèse unique, Université de Ouagadougou, $158 \mathrm{p}$.

White F. 1986. La végétation de l'Afrique. Mémoire accompagnant la carte de la végétation de l'Afrique Unesco/AETFAT/UNSO, OROTOM. $384 \mathrm{p}$. 